\title{
Impact of Cu Local Interconnect on LSI Performance
}

\author{
Takahiro Nagano $^{1}$, Shinichiro Kimura ${ }^{1}$ and Jin Onuki ${ }^{2}$ \\ ${ }^{1}$ Central Research Laboratory, Hitachi Ltd., Kokubunji 185-8601, Japan \\ ${ }^{2}$ Faculty of Systems and Technology, Akita Prefectural University, Honjo 015-0055, Japan
}

The effect of $\mathrm{Cu}$ local interconnect on intrinsic gate delay is analyzed. Beyond the 70-nm node, the intrinsic gate delay is limited by the local interconnect by taking account of electromigration in fine routings. Developments of materials and processes with improved electromigration for local interconnect are required.

(Received February 7, 2002; Accepted June 4, 2002)

Keywords: large scale integration (LSI), copper, local interconnect, gate delay

\section{Introduction}

An increase in clock frequency representative of LSI performance is attributed to a decrease in MOS transistor delay, which decreases by $S^{-1}$ where $S$ is the scaling factor $(S>1)$. In addition, the interconnect delay also affects the clock frequency. If all cross-sectional interconnect dimensions are scaled by the same factor as used for the transistors (ideal scaling), the delay time of global interconnect increases by $S^{2} S_{\mathrm{C}}^{2}$ where $S_{\mathrm{C}}$ is the scaling factor for the chip size $\left.\left(S_{\mathrm{C}}>1\right) .{ }^{1}\right) \mathrm{Cu}$ interconnect and low-k materials have been introduced to cope with the interconnect delay. ${ }^{2,3)} \mathrm{By}$ contrast, the influence of the local interconnect delay on LSI performance has been disregarded due to its constant value in the ideal scaling. The current density of local interconnect increases by $S$, which needs an improved electromigration with scaling. However, the electromigration failure lifetime decreases with decreasing line width. ${ }^{4}$ ) This means that the ideal scaling is inapplicable to the local interconnect. Thus, in this paper, new scaling scenarios for allowing the local interconnect to relax the current density with the same increase in packing density as that of transistors are proposed. The intrinsic gate delay, taking into account the local interconnect, is modeled and analyzed by numerical simulation.

\section{Scaling Scenario}

In the ideal scaling, all cross-sectional dimensions such as line width $W_{\mathrm{INT}}$, line spacing $W_{\mathrm{SP}}$, line thickness $H_{\mathrm{INT}}$, and insulator thickness $D_{\text {FOX }}$ decrease by the scaling factor $S^{1}$, while the current density $J_{\text {INT }}$ increases by $S^{1}$. In order to relax the current density $J_{\text {INT }}$, new scenarios of quasi-ideal and constant- $J$ scaling are proposed. The targets of the current density $J_{\text {INT }}$ are $S^{1 / 2}$ and $S^{0}$, respectively. Table 1 summarizes the effects of the above three scaling scenarios on local interconnect. The horizontal dimensions such as $W_{\mathrm{INT}}, W_{\mathrm{SP}}$, and line length $L_{\mathrm{INT}}$ are scaled by $S^{-1}$ to improve the overall packing density by the factor $S$. In contrast, the vertical dimensions $H_{\mathrm{INT}}$ and $D_{\mathrm{FOX}}$ are reduced by the same factor, where $H_{\mathrm{INT}}$ meets the targeted current density.

\section{Delay Modeling}

The effect of local interconnect on signal delay is evaluated not by the product of routing resistance $R_{\mathrm{INT}}$ and routing capacitance $C_{\mathrm{INT}}$, but by an equivalent circuit including transistors and local interconnect. Figure 1 shows an inverter-chain circuit that is a basic component of LSIs. The signal delay of the inverter-chain is evaluated using the equivalent circuit (Fig. 2), where $R_{\mathrm{TRS}}, C_{\mathrm{D}}$, and $C_{\mathrm{G}}$ represent the on-resistance, drain capacitance, and gate capacitance of MOS transistors,

Table 1 Scaling scenario for local interconnect.

\begin{tabular}{lccc}
\hline Parameter & $\begin{array}{c}\text { Ideal } \\
\text { scaling }\end{array}$ & $\begin{array}{c}\text { Quasi-ideal } \\
\text { scaling }\end{array}$ & $\begin{array}{c}\text { constant- } J \\
\text { scaling }\end{array}$ \\
\hline Current density, $J$ & $S^{1}$ & $S^{1 / 2}$ & $S^{0}$ \\
Line width, $W_{\mathrm{INT}}$ & $S^{-1}$ & $S^{-1}$ & $S^{-1}$ \\
Line space, $W_{\mathrm{SP}}$ & $S^{-1}$ & $S^{-1}$ & $S^{-1}$ \\
Line length, $L_{\mathrm{INT}}$ & $S^{-1}$ & $S^{-1}$ & $S^{-1}$ \\
Line thickness, $H_{\mathrm{INT}}$ & $S^{-1}$ & $S^{-1 / 2}$ & $S^{0}$ \\
Insulator thickness, $D_{\mathrm{FOX}}$ & $S^{-1}$ & $S^{-1 / 2}$ & $S^{0}$ \\
\hline
\end{tabular}

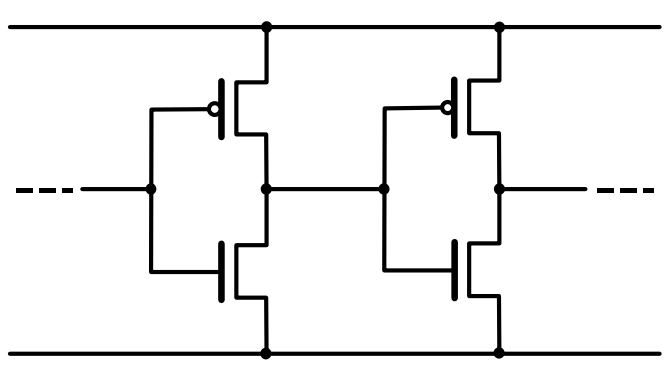

Fig. 1 Inverter-chain circuit.

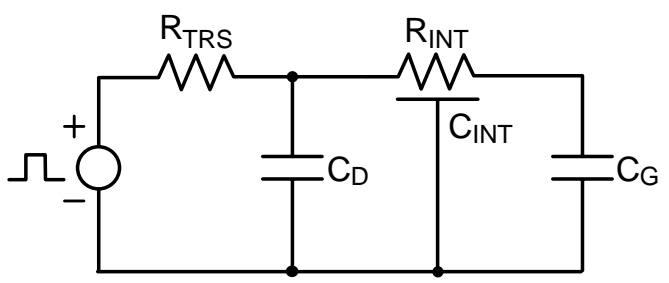

Fig. 2 Equivalent circuit. 
Table 2 Simulation Parameters in $\mathrm{nm}$.

\begin{tabular}{|c|c|c|c|c|c|c|c|}
\hline \multicolumn{2}{|c|}{ Technology node } & \multirow{2}{*}{$\begin{array}{l}180 \\
250\end{array}$} & \multirow{2}{*}{$\begin{array}{l}130 \\
180\end{array}$} & \multirow{2}{*}{$\begin{array}{l}100 \\
140\end{array}$} & \multirow{2}{*}{$\begin{array}{r}70 \\
100\end{array}$} & \multirow{2}{*}{$\begin{array}{l}50 \\
70\end{array}$} & \multirow{2}{*}{$\begin{array}{l}35 \\
50\end{array}$} \\
\hline & $W_{\mathrm{INT}}$ & & & & & & \\
\hline Ideal & $W_{\mathrm{SP}}$ & 250 & 180 & 140 & 100 & 70 & 50 \\
\hline \multirow[t]{3}{*}{ scaling } & $H_{\mathrm{INT}}$ & 300 & 216 & 168 & 120 & 84 & 60 \\
\hline & $D_{\text {FOX }}$ & 2500 & 1800 & 1400 & 1000 & 700 & 500 \\
\hline & $W_{\text {INT }}$ & & 180 & 140 & 100 & 70 & 50 \\
\hline \multirow{3}{*}{$\begin{array}{l}\text { Quasi-ideal } \\
\text { scaling }\end{array}$} & $H_{\mathrm{INT}}$ & & 255 & 224 & 190 & 159 & 134 \\
\hline & $D_{\text {FOX }}$ & & 2121 & 1871 & 1581 & 1323 & 1118 \\
\hline & $W_{\text {INT }}$ & & 180 & 140 & 100 & 70 & 50 \\
\hline \multirow{2}{*}{$\begin{array}{l}\text { Constant- } J \\
\text { scaling }\end{array}$} & $W_{\mathrm{SP}}$ & & 180 & 140 & 100 & 70 & 50 \\
\hline & $H_{\mathrm{INT}}$ & & 300 & 300 & 300 & 300 & 300 \\
\hline
\end{tabular}

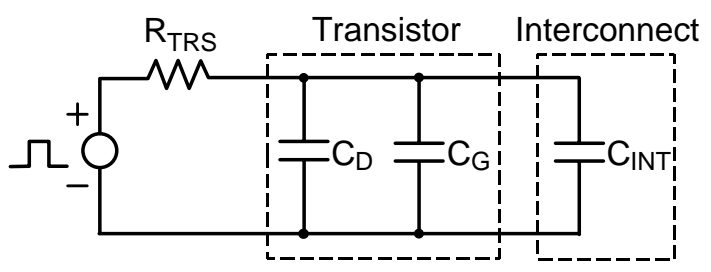

Fig. 3 Interconnect model for signal delay evaluation.

respectively. Figure 3 gives an approximation for the signal delay by considering a negligible value of $R_{\mathrm{INT}}$ to $R_{\mathrm{TRS}}$. Thus, the signal delay is simply evaluated by the product of $R_{\mathrm{TRS}}$ and parasitic capacitance $\left(C_{\mathrm{TRS}}\left(=C_{\mathrm{D}}+C_{\mathrm{G}}\right)+C_{\mathrm{INT}}\right)$.

\section{Results and Discussion}

\subsection{Routing capacitance}

Figure 4 shows a local interconnect model to evaluate the routing capacitance. Routings $\mathrm{INT}_{\mathrm{L}}$ and $\mathrm{INT}_{\mathrm{R}}$ are arranged in parallel with routing $\mathrm{INT}_{\mathrm{C}}$. All the routings are formed above the silicon-substrate, and a field-oxide insulator with a thickness of $D_{\text {FOX }}$ is in-between. The value of routing capacitance is obtained by using CLEVER, ${ }^{5)}$ a three-dimensional electrostatic field solver. Table 2 lists simulation parameters.

Dependence of routing capacitance per unit length on technology node $X_{\mathrm{TN}}$, plotted in Fig. 5, is approximated by $X_{\mathrm{TN}}^{0}$, $X_{\mathrm{TN}}^{-1 / 3}$, and $X_{\mathrm{TN}}^{-2 / 3}$, for the ideal scaling (IS), quasi-ideal scaling (QS), and constant- $J$ scaling (CS), respectively. Thus, the routing capacitance $C_{\mathrm{INT}}$ for IS, QS, and CS decreases by $S^{-1}, S^{-2 / 3}$, and $S^{-1 / 3}$, respectively, by considering both $X_{\mathrm{TN}}$ and line length $L_{\mathrm{INT}}$ decrease by $S^{-1}$. Therefore, relaxation of the current density increases the routing capacitance. The transistor capacitance $C_{\mathrm{TRS}}$ decreases by $S^{-1}$, thus suggesting that the routing capacitance $C_{\text {INT }}$ will limit the intrinsic gate delay in shorter technology nodes.

\subsection{Scaling of $\boldsymbol{C}_{\mathrm{TRS}}$ and $\boldsymbol{C}_{\mathrm{INT}}$}

A quantitative comparison between the transistor capacitance $C_{\mathrm{TRS}}$ and routing capacitance $C_{\mathrm{INT}}$ is graphed in Fig. 6 , with the technology node $X_{\mathrm{TN}}$ of 180 -nm chosen as a standard. The following device dimensions are expected from the

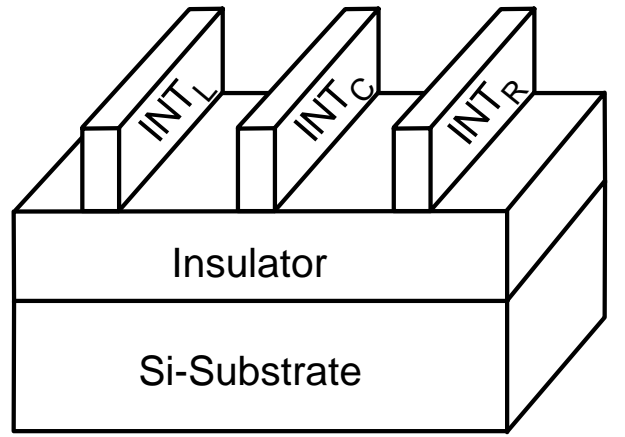

Fig. 4 Local interconnect model to evaluate routing capacitance.

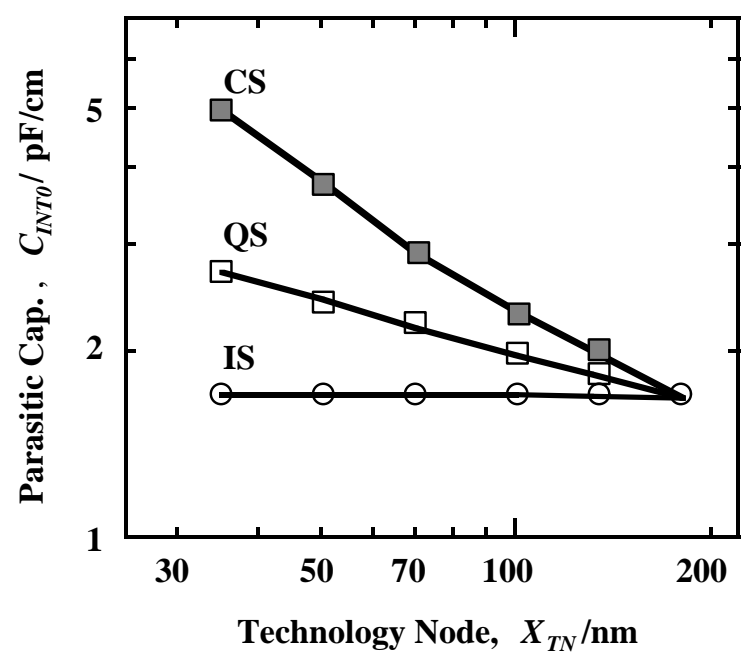

Fig. 5 Routing capacitance per unit length vs. technology node for ideal scaling (IS), quasi-ideal scaling (QS), and constant- $J$ scaling (CS).

references: ${ }^{6,7)}$ gate length $L_{\mathrm{G}}$ of $140 \mathrm{~nm}$, gate width $W_{\mathrm{G}}$ of $700 \mathrm{~nm}$, drain junction area of $350 \times 700 \mathrm{~nm}^{2}$, effective gate oxide thickness of $3 \mathrm{~nm}$, and typical interconnect length $L_{\mathrm{INT}}$ of 3,500 nm (around 20 times of $X_{\mathrm{TN}}$ ). If constant- $J$ scaling is applied, curves for $C_{\mathrm{TRS}}$ and $C_{\mathrm{INT}}$ intersect at the technology node of $70-\mathrm{nm}$. This means that the intrinsic gate delay is limited mainly by the local interconnect beyond the 70-nm node, and thus the constant- $J$ scaling scenario should not be applied. Whereas the current density $J_{\text {INT }}$ increases by $S^{1 / 2}$ 


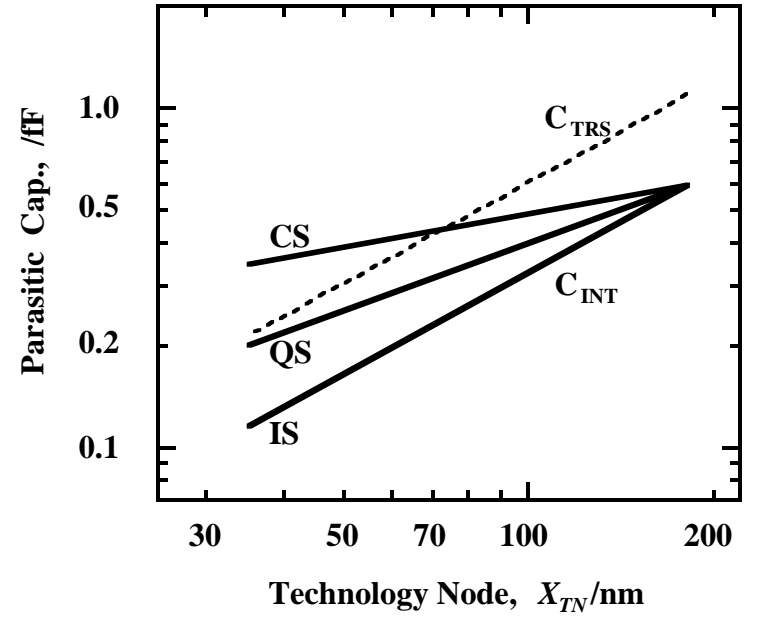

Fig. 6 Parasitic capacitance $C_{\mathrm{TRS}}$ and $C_{\mathrm{INT}}$ vs. technology node for ideal scaling (IS), quasi-ideal scaling (QS), and constant- $J$ scaling (CS). Interconnect capacitance is evaluated at $L_{\mathrm{INT}}$ of 20 times of $X_{\mathrm{TN}}$.

and $S^{1}$ for QS and IS, respectively, developments of materials and processes with an increase in electromigration by more than $S^{1 / 2}$ are indispensable.

\section{Conclusion}

The influence of a $\mathrm{Cu}$ local interconnect on signal delay in connection with its electromigration has been analyzed.
The current densities in the ideal, quasi-ideal, and constant$J$ scaling scenarios decrease by $S^{1}, S^{1 / 2}$, and $S^{0}$, whereas the routing capacitances increase by $S^{-1}, S^{-2 / 3}$, and $S^{-1 / 3}$, respectively. Beyond the $70-\mathrm{nm}$ node, the intrinsic gate delay is limited by the local interconnect in the constant- $J$ scaling. This requires developments of interconnect materials and processes with improved electromigration.

\section{Acknowledgements}

The authors would like to thank Y. Sasaki and Dr. H. Mizuno for providing insight into topics discussed in this paper.

\section{REFERENCES}

1) K. C. Saraswat and F. Mohammadi: IEEE J. Sold-State Circuits SC-17 (1982) 275-280.

2) D. Edelstein et al.: IEDM Tech. Dig. (1997) 773-776.

3) S. Venkatesan et al.: IEDM Tech. Dig. (1997) 769-772.

4) C-K Hu, K. Y. Lee, L. Gignac and R. Carruthers: Thin Solid Films 308309 (1997) 443-447.

5) CLEVER User's Manual, SILVACO International, Version 2.0 May 1999.

6) International Technology Roadmap for Semiconductors 2000 Update, by the Semiconductor Industry Association \& SEMATECH.

7) S. Borkar: IEEE Micro (1999) 23-29. 